\section{Approches économiques du tabagisme}

Michel Grignon, Bertrand Pierrard

> Afin de statuer sur la légitimité de l'intervention des pouvoirs publics dans les comportements de consommation de tabac, les économistes ont tenté de modéliser la dépendance tabagique. Deux modèles théoriques sont proposés à ce jour: le modèle de dépendance rationnelle, qui formalise un individu aliénant rationnellement sa liberté future de consommation, et le modèle de capital santé, qui formalise un individu arbitrant entre un plaisir et son espérance de vie. Les tests de pertinence de ces modèles théoriques ont une importance pratique en matière de recommandations de politique publique de lutte contre le tabac: si l'hypothèse de dépendance rationnelle est vérifiée, des politiques de prix annoncées, crédibles et régulières constituent le moyen le plus efficace de lutte contre le tabagisme. En revanche, si les fumeurs sont piégés dans la dépendance, augmenter les prix est non seulement peu efficient, mais aussi inutilement cruel pour les fumeurs; on se tournera alors vers des politiques de dissuasion d'initiation ou d'aide au sevrage. Sans toutefois être exempt de problème, il semble à l'analyse de la littérature que le modèle de capital santé rende mieux compte des faits empiriques observés à propos du tabagisme que le modèle de dépendance rationnelle. <

Fumer du tabac est dangereux à long terme pour la santé et entraîne un risque très important de dépendance $^{l}$. Les économistes s'appuient sur trois termes de cette définition («dangereux», «à long terme», «dépendance $\left.{ }^{l} »\right)$ pour évaluer la légitimité des politiques publiques de lutte contre le tabagisme. Le dan-

${ }^{1} \varepsilon$ n cas de dépendance à une substance ou à un comportement, les choix actuels de consommation de l'individu dépendent de ses choix de consommation passés: le fumeur dépendant continue de fumer non pas en raison du plaisir apporté, mais plutôt par crainte de la gêne occasionnée par une réduction de sa consommation. La dépendance introduit une dissymétrie dans la relation entre volume consommé et plaisir : passer de $\mathrm{N}$ à $\mathrm{N}-\mathrm{n}$ unités entraine plus de peine que passer de $\mathrm{N}-\mathrm{n}$ à $\mathrm{N}$ n'a procuré de plaisir.

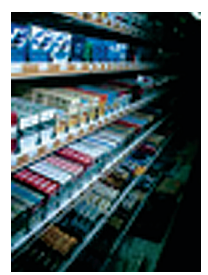

ger ne suffit pas en luimême à justifier l'intervention: si l'on croit que
Institut de recherche et de documentation en économie de la santé (IRDES), 10 , rue Vauvenargues, 75018 Paris, France. grignon@irdes.fr le consommateur est rationnel (hypothèse partagée par la plupart des économistes, moyennant des nuances détaillées ci-dessous), personne ne peut l'empêcher de retirer plaisir d'un danger si celui-ci ne pénalise personne d'autre que lui ${ }^{2}$. En revanche, le fait que la réalisation du risque soit reportée très longtemps après la consommation peut légitimer l'intervention publique: le consommateur peut être rationnel, mais manquer d'information sur le futur, ou négliger les états futurs au profit du plaisir présent (on parle de préférence forte pour le présent, attitude psychologique généralement attribuée aux jeunes). La puissance publique est alors légitime pour donner plus de poids au futur ou plus d'informations sur le futur, soit directement (par un affichage sur les paquets, par exemple), soit indirectement, en renchérissant artificiellement le tabac via l'augmentation des taxes. De même, le fait que la cigarette rende le fumeur dépendant peut justifier l'intervention publique si l'individu ignore qu'il sera dépendant (ou à quel degré) avant de commencer à fumer; là encore, l'individu peut être découragé soit par une augmentation du prix du tabac, soit, même si c'est encore rare, par une diffusion de l'information sur le risque de dépendance.

\section{Modèles de dépendance}

Les deux modèles proposés par les économistes reposent sur l'hypothèse classique de la théorie micro-économique: les comportements effectifs d'un individu sont le

\footnotetext{
${ }^{2}$ Ce dernier point a donné lieu à une abondante littérature, en premier lieu sur le coût social que les maladies des fumeurs imposent aux non-fumeurs, et plus récemment sur le tabagisme passif [1].
} 
fruit de décisions rationnelles visant à maximiser sa satisfaction, une décision étant jugée rationnelle lorsque l'individu tient compte des conséquences qu'elle peut entraîner. Pour maximiser sa satisfaction, l'individu alloue au mieux de ses préférences le budget dont il dispose à différents biens et services. Le premier modèle, dû à G.S. Becker et K.M. Murphy [2], appelé « dépendance rationnelle », formalise un individu décidant rationnellement de débuter une consommation engendrant une dépendance, mais néglige pour ce faire la prise en compte par l'individu des conséquences sanitaires (notamment sur la durée de vie) de cette consommation; à l'inverse, le second modèle, explicité entre autres par Suranovic et al. [3], appelé « capital santé », met en scène un individu prévoyant les conséquences en années de vie perdues de la consommation tabagique, mais négligeant la pénibilité de l'arrêt.

\section{Dépendance rationnelle}

G.S. Becker et K.M. Murphy ne s'intéressent pas spécifiquement au tabagisme, si ce n'est comme test d'un modèle dynamique de la prise de décision. Dans un modèle dynamique, un individu prend à chaque date la meilleure série possible de décisions futures (jusqu'à sa mort) compte tenu de son environnement (essentiellement les prix relatifs) à cette date. Par la suite, il ne se conformera peut-être pas à ce programme si l'environnement change; en revanche, si l'environnement reste inchangé, il n'aura aucune raison de dévier de ce programme. Décider de commencer à fumer est bien une décision qui change les choix futurs, ne serait-ce que celui de fumer ou non, en raison de la dépendance.

R.A. Pollak [4] avait proposé de formaliser ce type de décisions dynamiques en faisant l'hypothèse que le fait de fumer à une période $t$ changeait les préférences de l'individu pour les périodes suivantes. Cette formalisation ayant été jugée non conforme à l'individualisme méthodologique ${ }^{3}$ [5], G.S. Becker et K.M. Murphy ont proposé un modèle où l'individu conserve les mêmes préférences tout au long de sa vie; mais au lieu de préférences «simples» pour les caractéristiques intrinsèques et observables des biens et des services, ils proposent le concept de «métapréférences », incluant des éléments non observables liés à l'histoire ou au contexte des biens et des services. Dans la situation d'une consommation de bien avec dépendance, comme c'est le cas au cours du tabagisme, ces métapréférences incluent le «stock» de consommations antérieures de cigarettes: si les individus ne changent pas de préférences, le stock accumulé de consommations antérieures les pousse à prendre «rationnellement», vis-à-vis de leur consommation courante, des décisions différentes de celles qu'ils auraient

${ }^{3}$ L'individualisme méthodologique postule l'existence d'un homo œeconomicus dont une des caractéristiques est l'immuabilité des préférences dans le temps, celles-ci étant insensibles au contexte dans lequel évolue l'individu comme à son histoire. Le comportement est, quant à lui, sensible à l'environnement: à préférences constantes, des prix différents, par exemple, engendrent des choix différents. prises en l'absence de ce stock. Cette formalisation assimile le tabagisme à un investissement: le plaisir retiré de la consommation de tabac actuelle (et, d'ailleurs, la peine liée à la nonconsommation actuelle) dépend d'une compétence à apprécier le tabac ${ }^{4}$ (ou d'une difficulté à s'en passer), elle-même liée au stock des consommations passées.

La stabilité de ses préférences permet bien à l'individu de prévoir à chaque période l'ensemble de ses satisfactions présentes et futures et le conduit donc à un plan de maximisation de l'utilité stable dans le temps. C'est vraiment là que se trouve l'apport principal, sur le plan théorique, du modèle de dépendance rationnelle. D'un point de vue formel, le consommateur «dépendant» est équivalent au consommateur classique: il maximise la somme sur sa vie entière du plaisir retiré, à chaque période, de sa consommation de tabac (plaisir lui-même lié au stock de compétence tabagique accumulée) et de la consommation d'un autre bien pour lequel on ne suppose aucun effet de dépendance (c'est-à-dire tous les biens sauf la cigarette). Le modèle de dépendance rationnelle propose un lien entre la consommation présente de tabac et le stock de tabagisme accumulé: sa principale prédiction est l'existence d'une consommation d'équilibre telle que la consommation courante de tabac entretient, sans l'augmenter, la compétence accumulée à apprécier le tabac.

Il prévoit aussi que la consommation d'équilibre dépend de facteurs exogènes, comme le prix des cigarettes, mais aussi de facteurs individuels, comme la préférence pour le présent: les individus les plus «impatients », qui, d'un point de vue technique, ont un taux d'actualisation ${ }^{5}$ supérieur, accordent moins d'importance aux événements futurs, et notamment à la dépendance; ils commenceront donc à fumer plus volontiers, puisque le risque lié à la dépendance compte moins pour eux. De plus, comme une autre prédiction de l'analyse du comportement par les métapréférences est que les individus les plus impatients font moins d'études, G.S. Becker et K.M. Murphy en déduisent que leur modèle «prédit » correctement le taux de fumeurs supérieur observé chez les moins diplômés.

Enfin, ce modèle avance qu'il n'y a pas pas de solution d'équilibre quand la dépendance est trop forte, d'où l'existence de conduites apparemment irrationnelles, comme les conduites quasi suicidaires de certains toxicomanes. Le caractère addictif d'un bien pourrait alors être déduit de la proportion observée d'individus montrant des consommations toujours croissantes et sans équilibre.

${ }^{4}$ II s'agit là de la compétence accumulée à apprécier le tabac, une définition « positive » de la dépendance.

${ }^{5}$ Actualiser une valeur, c'est tenir compte de la dépréciation d'un bien (ou d'un service) en fonction de sa date de disponibilité. On applique à la valeur intrinsèque du bien ou service le taux d'actualisation (montant de cette dépréciation) pour obtenir l'équivalent-valeur de ce bien par rapport aux biens disponibles maintenant. Théoriquement, un individu rationnel situé dans un environnement certain ne devrait avoir aucune préférence entre un bien aujourd'hui et le même bien demain. Une concession de ce modèle de l'individu rationnel à la psychologie (parfois justifiée par l'existence d'incertitude sur le futur) est d'admettre que l'on préfère le même bien (ou la même quantité d'argent) aujourd'hui à demain. 
Ce modèle de décision dynamique sur la durée de vie ne permet pas de considérer l'impact du tabagisme sur l'espérance de vie. II autorise tout au plus une simulation des effets néfastes éventuels du bien addictif par son effet négatif sur la productivité de l'individu, donc sur son revenu futur.

\section{Modèle de capital santé}

Avant sa formalisation par Suranovic et al. [3], ce modèle, appliqué ou non à la consommation de tabac, a fait l'objet d'études et de développements par différents auteurs [6-10]. Dans le modèle de capital santé, les individus sont supposés sensibles à leur état de santé, ou, plus précisément, à leur espérance de vie, et arbitrent rationnellement entre certains plaisirs pathogènes (consommation de tabac et d'alcool, vie sédentaire) et l'utilité apportée par des années de vie supplémentaires. Une telle modélisation fait intervenir un individu somme toute plus proche de l'intuition que le calculateur à horizon infini du modèle de dépendance rationnelle: n'est-il pas naturel de pondérer les efforts nécessaires à l'obtention d'une forte longévité par le coût engendré par ces efforts? Plus prosaïquement, notre individu se demande si une vie courte, mais remplie de plaisirs, n'est pas préférable à une vie longue, mais austère.

Malgré une certaine proximitéb, les modèles de dépendance rationnelle et de capital santé n'en sont pas moins irréconciliables: on pourrait certes imaginer un modèle théorique dans lequel l'individu tiendrait compte simultanément de son capital santé et de son capital addictif, mais, outre la difficulté analytique de traiter un tel modèle, on ne pourrait trouver de tests empiriques permettant de départager les effets de ces deux variables, dans la mesure où elles sont inobservables.

Dans l'approche du capital santé, le piège de la dépendance est donc ignoré par le consommateur, et l'on retrouve plus ou moins le modèle à changements de préférences de R.A. Pollak: l'individu ayant fumé longtemps éprouve de la peine à passer à un niveau de consommation inférieur, qui lui apporterait pourtant satisfaction s'il n'avait jamais fumé. Cette peine peut être interprétée comme un coût [3], de quelque forme que ce soit: on peut imaginer des coûts convexes (plus on diminue, plus c'est difficile, cas d'une dépendance faible), concaves (les premiers pas sont les plus difficiles, cas d'une dépendance forte) ou sigmoïdes (convexes pour des petites diminutions, puis concaves).

Le modèle fonctionne alors comme suit: à chaque période de sa vie, l'individu arbitre sur un nombre de cigarettes idéal en comparant le plaisir apporté par la cigarette et la perte estimée en espérance de vie. Ce nombre idéal de cigarettes fumées engendre cependant un coût lié à la dépendance, et l'individu ne pourra donc pas «s'offrir » la décision optimale au regard de l'arbitrage initial. Un tel modèle rend compte assez simple-

${ }^{6}$ Les deux modèles sont en effet proches dans leur conception du consommateur, le modèle de Suranovic et al. [3] attribuant un rôle important à l'état de santé attendu, qui est aussi une « consommation » non directement observable. ment de plusieurs comportements de fumeurs. II existe ainsi des fumeurs malheureux que le coût de l'arrêt empêche de réaliser un arbitrage optimal et qui continuent de fumer alors que le plaisir des cigarettes fumées est moindre que la désutilité liée à la diminution d'espérance de vie; par ailleurs, l'arrêt total de la consommation de cigarettes peut être une stratégie rationnelle, pour peu que le coût augmente moins vite que le gain tiré de l'amélioration de l'espérance de vie; enfin, un tel modèle rend compte assez naturellement de l'augmentation avec l'âge de l'envie d'arrêter sa consommation de tabac: I'individu se rapproche en effet du moment où les conséquences sanitaires du tabagisme sont susceptibles de se faire sentir.

\section{Tests empiriques}

L'approche classique en économie consiste à tester une théorie en déduisant des conclusions (hors des hypothèses de construction de la théorie) et en testant l'adéquation de ces conclusions avec des observations économétriques. Depuis quelques années, cependant, une économie expérimentale se développe, qui vise à tester les hypothèses psychologiques fondamentales des modélisations, notamment celles portant sur les préférences des individus.

Les deux approches décrites ci-dessus sont, du point de vue des tests empiriques, dans des situations opposées: la dépendance rationnelle a donné lieu à une littérature abondante sur ses prédictions, notamment sur le lien entre prix anticipés et consommation de cigarettes, mais ses fondements psychologiques, notamment l'aptitude des individus à prévoir leur situation future, ont été peu testés. À l'inverse, les mécanismes psychologiques laissant supposer que l'individu tient compte de son espérance de vie quand il décide ou non de fumer ont été nettement plus testés que les prédictions du modèle de capital santé: à notre connaissance, seules deux études [10, 11] proposent une validation empirique de ses conclusions.

\section{Fondements psychologiques}

Chacun des deux modèles repose sur un scénario psychologique: le consommateur de la dépendance rationnelle sait se projeter dans l'avenir, et celui du capital santé sait arbitrer entre risque sanitaire futur et plaisir immédiat. De tels scénarios sont testables et on peut chercher à savoir, par enquête, lequel est le plus conforme aux mécanismes «réels» à l'œuvre dans la population des consommateurs. Ces tests par enquêtes semblent tous rejeter l'hypothèse de dépendance rationnelle (pour revue, voir [1]), et soutenir plus ou moins celle de capital santé.

En premier lieu, les enquêtes longitudinales auprès de fumeurs débutants montrent que les individus sous-estiment leur propre dépendance: en moyenne, le taux d'arrêt lors de la seconde interrogation, qui a lieu un an après la première, est beaucoup plus faible que ne laissait prévoir le degré de dépen- 
dance estimé par les enquêtés lors de leur première interrogation; par ailleurs, ce que pense un individu de sa dépendance n'a aucune influence sur la probabilité qu'il arrête de fumer dans l'année, tout au moins chez les jeunes.

En deuxième lieu, les enquêtes directes auprès des fumeurs, comme celles menées auprès des familles de fumeurs ou des médecins, montrent qu'il existe des fumeurs « accrochés » et malheureux qui souhaiteraient arrêter, mais rechutent. Ces fumeurs sous-estiment le risque de maladie lié au tabac, tout en surestimant leur capacité à s'arrêter [12].

Ensuite, il apparaît que les individus ne négligent pas le risque lié au tabagisme (ils auraient même tendance à le surestimer) et, surtout, qu'ils en tiennent compte dans leur comportement vis-à-vis du tabac. Des tests menés à Taïwan [13], au sein d'une population pour laquelle les messages de prévention liés au tabac sont assez récents, montrent que le degré d'information diminue la probabilité d'être un fumeur régulier, toutes choses égales par ailleurs ${ }^{7}$.

Enfin, les données d'enquêtes contredisent les fondements psychologiques du modèle de dépendance rationnelle, notamment en ce qui concerne la capacité des individus à maximiser leur satisfaction sur l'ensemble de leur vie. Rappelons que, selon G.S. Becker et K.M. Murphy, l'individu ne change pas ses décisions optimales tant que les prix (ou son revenu) ne varient pas: d'après leur hypothèse, l'individu verra le futur comme il le voit maintenant, l'actualisation entre deux périodes futures n'étant pas appelée à changer à mesure qu'elles se rapprochent du présent. Pourtant, si l'on demande à des individus de faire des choix entre diverses situations futures, la plupart d'entre eux ont une préférence biaisée pour le présent: leurs choix varient avec l'éloignement de ces deux dates par rapport au temps présent; concrètement, alors que deux dates distantes d'un an apparaissent équivalentes (pas d'actualisation entre elles) quand elles sont éloignées de plusieurs années par rapport au présent, le consommateur ne leur attribue plus la même valeur (actualisation non nulle) quand l'une d'elles est le présent.

\section{Tests sur les prédictions}

Dépendance rationnelle ou capital santé s'accordent pour «prédire » que le tabagisme laisse une part non négligeable aux choix individuels, retrouvant d'ailleurs des résultats d'observations déjà réalisées par les sociologues à propos de la consommation de substances illégales [14]; les variables de prix ou d'information (diplôme) jouent donc un rôle classique en économie du consommateur dans ces deux théories. En fait, on peut à peine parler de prédictions, puisque ces modèles ont été construits pour reproduire les régularités observées du comportement tabagique.

\section{Prédictions du modèle de dépendance rationnelle}

${ }^{7}$ Les tests ont contrôlé le biais lié au fait que les individus qui ne peuvent s'arrêter de fumer préfèrent occulter les messages de prévention.
Si l'hypothèse de dépendance rationnelle est vraie, le prix anticipé des cigarettes doit jouer un rôle décisif dans la consommation de tabac.

Un lien statistique significatif a ainsi été retrouvé entre le prix futur du tabac et sa consommation présente, ce qui validerait le modèle de dépendance rationnelle [15]. Ce résultat a cependant été critiqué, notamment parce que le test était mené sur des données agrégées (ventes de cigarettes par État). Ces mêmes données pouvaient d'ailleurs valider un modèle alternatif dans lequel les fabricants de tabac s'entendaient sur les prix (configuration d'oligopole) et augmenteraient le prix unitaire en réponse à une baisse de la consommation [16].

Un test mené auprès de femmes enceintes ${ }^{8}$ [17] a observé un effet d'anticipation du prix à court terme sur la consommation de tabac, ce qui suggère que les individus sont capables de se projeter dans un futur proche (il faut toutefois noter que cette étude concernait une population particulière, par ailleurs plus sensibilisée que la population générale aux méfaits du tabac). À ce stade, les résultats obtenus sur les comportements d'arrêt ou de réduction de la consommation n'infirmaient pas l'hypothèse de la dépendance rationnelle. Toutefois, ce modèle devait encore subir un test, concernant le fait de commencer à fumer (l'« initiation tabagique »). En effet, le modèle théorique fait jouer un rôle décisif à la compétence acquise par la pratique, et ne fournit donc aucun facteur décisif permettant de savoir ex ante qui sera fumeur et qui restera abstinent au sein d'une population soumise aux mêmes prix présents et anticipés. Dans le modèle de base, l'initiation est fonction d'un phénomène purement aléatoire (n’obéissant donc à aucune régularité collective), le stress lié à des événements familiaux affectant l'individu. Si le modèle est valide, le seul facteur observable dont dépend l'initiation tabagique est le prix du tabac. Une étude exploitant des données tranversales obtenues sur plusieurs générations d'individus a exploré la relation entre le fait de commencer à fumer, ainsi que l'âge d'initiation, et le prix du tabac à l'époque où l'individu avait 15 ans et 18 ans (approximation du prix anticipé) [18]. Aucun effet significatif du prix du tabac n'a été retrouvé, alors que des régularités collectives ont été mises en évidence, dont le modèle de dépendance rationnelle ne peut rendre compte: ainsi, par exemple, les adolescents blancs fument plus souvent et commencent plus jeunes que leurs équivalents d'origine afro-américaine ou hispanique?

\section{Prédictions du modèle de capital santé}

Si l'hypothèse de capital santé est vraie, la probabilité de réussite d'un arrêt de la consommation dépend de manière cruciale de la quantité de tabac fumée.

L'enjeu des études réalisées $[10,11]$ a essentiellement été de

\footnotetext{
${ }^{8}$ Le suivi exhaustif des grossesses sur le territoire américain contient une question sur la consommation tabagique et permet donc de disposer de données suffisamment nombreuses pour mesurer l'évolution de la consommation entre l'annonce d'une hausse du prix du tabac et son entrée en vigueur effective.

${ }^{9}$ Dans les enquêtes individuelles américaines, il s'agit de caractéristiques autodéclarées.
} 
tester l'influence du comportement tabagique sur la probabilité de réussir un sevrage. Le modèle prédit en effet que les plus gros fumeurs ont, toutes choses égales par ailleurs, une difficulté supérieure à réussir l'arrêt: ce résultat a bien été retrouvé dans les estimations. Celles-ci ont consisté à expliquer, dans un premier temps, la décision de fumer ou non (équation dite de participation), puis, dans un second temps, le volume consommé par les fumeurs (consommation conditionnelle) en tenant compte des liens entre ces deux équations au niveau individuel (modèle double hurdle). Les résultats ont permis de mettre en évidence une relation inverse entre la décision de participer et la consommation conditionnelle: un facteur expliquant une probabilité plus faible d'être fumeur (par exemple un diplôme élevé) explique aussi que les fumeurs ayant cette caractéristique fument plus.

Les estimations ont par ailleurs abouti à un résultat inattendu: plus on a commencé tard, moins on est susceptible d'arrêter, toutes choses égales par ailleurs. Tout se passe comme si le fumeur voulait assurer une durée minimum dans l'état, indépendamment de son âge d'initiation; or cette observation n'est ni prédite, ni expliquée par un modèle de choix rationnel des plaisirs immédiats et des peines futures.

\section{Test de stabilité des préférences des individus}

Si les préférences dépendent du contexte (groupes de pairs, environnement...), le modèle de capital santé est plus probable que le modèle de dépendance rationnelle. En effet, si le modèle de capital santé admet assez facilement que les préférences évoluent avec la situation dans laquelle se trouve l'individu ${ }^{10}$, la stabilité et l'universalité des métapréférences sont, à l'inverse, des postulats de base du modèle de dépendance rationnelle, dans lequel l'individu doit faire des choix cohérents sur l'ensemble du cycle de vie. En fait, la plupart des résultats empiriques remettent en question l'hypothèse de stabilité et d'universalité des préférences vis-à-vis du tabac.

Le point le plus important est ici la mesure de l'influence de la publicité ou des groupes de pair. En ce qui concerne la publicité, la question est de savoir si la quantité de publicité influence la consommation tabagique d'ensemble ou seulement les parts de marché des différentes marques au sein d'un volume stable. Les études $[19,20]$ montrent que la publicité influence positivement le volume total consommé, et a donc une incidence sur la préférence à l'égard du tabac.

$\varepsilon n$ ce qui concerne l'effet des groupes de pairs, la difficulté d'analyse vient du fait que la corrélation statistique n'indique pas nécessairement un lien causal d'influence: si l'individu

\footnotetext{
${ }^{10}$ Les auteurs du modèle de capital santé ne se prononcent pas véritablement sur le point de savoir si leur individu représentatif doit avoir des préférences dépendantes du contexte. On peut seulement lire dans leur modèle que l'individu ajuste ses décisions à chaque période en fonction de préférences qui sont affectées par son passé; on peut en déduire que ce modèle supporterait plus facilement des préférences dépendantes du contexte que celui de la dépendance rationnelle, qui les rejette explicitement (et a même été construit pour formaliser un comportement dynamique sans effet du contexte sur les préférences).
}

choisit ses pairs notamment sur la base de la similitude des comportements tabagiques, la corrélation positive des comportements ne signifiera nullement l'existence d'une influence. En contrôlant ce biais potentiel par une technique de variable instrumentale, il a pu être conclu sans ambiguïté à l'existence d'un effet d'influence du groupe de pairs sur la décision de commencer à fumer [21].

\section{Conclusions}

Actuellement, le modèle de capital santé est celui qui rend le mieux compte des faits empiriques observés dans l'étude du tabagisme: la mise en évidence d'un lien entre degré de dépendance et capacité à réussir le sevrage accrédite l'idée de piège lié au tabagisme, tandis que le rôle des influences externes sur les préférences individuelles, notamment au moment de l'initiation, semble contredire l'idée que les individus font des choix cohérents en début de carrière tabagique.

Pour autant, le modèle de capital santé n'est pas exempt de problèmes. II ne rend pas compte, par exemple, de toutes les régularités constatées, notamment de l'influence propre de la durée de la carrière tabagique sur la probabilité d'arrêter. Par ailleurs, il remet en cause la cohérence et la force du modèle orthodoxe du consommateur en économie: alors que ce dernier relie les comportements à des éléments exogènes comme les prix ou le revenu individuel, le modèle de capital santé nécessite la prise en compte d'effets plus complexes du contexte sur les préférences individuelles. $\diamond$

\section{SUMMARY}

\section{Economic approaches of tobacco consumption}

In order to assess regulator's legitimacy in discouraging people from smoking, economists model tobacco addiction. In this paper, we review the extent economic literature about tobacco consumption and tobacco addiction. Two main approaches are singled out. In the first section, we detail the main characteristics of the two main theoretical models: rational addiction on one hand, which stylises individual choosing now to give up their future liberty to choose whether or not to smoke; and health capital on the other hand, following which individuals trade off between current pleasure and life expectancy. Second section gives account of the empirical tests of these two theories, before a last section briefly discusses and concludes. We show that the health capital theory seems to pass the test better than the rational addiction model. It is important to discriminate between these two models, from a political economy point of view. Were rational addiction true, a constant and strong increase of taxes on tobacco would be the soundest policy to curb consumption. On the contrary, if smokers are trapped into addiction and can't stop without pain, then tax increase is imposing too much strain on them, for no result. An efficient policy would be to subsidise quitting programmes, or to help 
smokers reducing their consumption through partial prohibition

(in public transports for instance). $\diamond$

\section{RÉFÉRENCES}

1. Dupilet C, Grignon M, Pierrard B. Modèles économiques et politiques de lutte contre le tabagisme. Rapport $n^{\circ} 1381$. Paris: CREDES, 2002: $130 \mathrm{p}$.

2. Becker GS, Murphy KM. A theory of rational addiction. J Polit $\varepsilon$ con 1988 ; 96: 675-700.

3. Suranovic SM, Goldfarb RS, Leonard TC. An economic theory of cigarette addiction. J Health Econ 1999; 18: 1-29.

4. Pollak RA. Habit formation and dynamic demand function. J Polit Econ 1970; 78: $745-63$.

5. Stigler GJ, Becker GS. De gustibus non est disputandum. Am Econ Rev 1977 ; 67: 76-90.

6. Grossman M. On the concept of health capital and the demand for health. J Polit Econ 1972; 80: 223-55.

7. Fuchs VR. Time preference and health: An exploratory study. In: Fuchs VR, ed. Econ Asp Health. New York: Columbia University Press, 1982: 93-120.

8. Viscusi WK. Age variations in risk perception and smoking decision. Rev Econ Stat $1991 ; 73: 577-88$.

9. Kenkel DS. Health behaviour. Health knowledge and schooling. J Polit Econ 1991; 99: 287-305.

10. Jones AM. Health, addiction, social interaction and the decision to quit smoking. J Health Econ 1994; 13 : 93-110.
11. Yen ST, Jones AM. Individual cigarette consumption and addiction: a flexible limited dependent variable approach. Health Econ 1996; 5: 105-17.

12. Emery $S$, Gilpin $\varepsilon A$, Ake $C$, et al. Characterizing and identifying «hard-core » smokers: Implications for further reducing smoking prevalence. Am J Public Health 2000; $90: 387-94$

13. Liu BQ, Peto R, Chen ZM, et al. Emerging tobacco hazards in China: 1 . Retrospective proportional mortality study of one million deaths. Br Med J 1998; 317: 1411-22.

14. Ogien A. Sociologie de la déviance et usages de drogues. Une contribution de la sociologie américaine. Document du Groupement de recherche «Psychotropes, politique et société », $n^{\circ} 5$. Paris: CNRS, 2000:64 p.

15. Becker GS, Grossman M, Murphy KM. Rational addiction and the effect of price on consumption. Am Econ Rew 1991; 81: 237-41.

16. Showalter MH. Essays in applied econometrics. Essay III: Monopoly behavior with intertemporal demands. Cambridge (MA): Massachusetts Institute of Technology, 1991.

17. Gruber J, Köszegi B. Is addiction «rational»? Theory and evidence. Working Paper, $n^{\circ} w 7507$. National bureau of economic research (NBER), 2000.

18. Douglas $S$, Hariharan $G$. The hazard of starting smoking: estimates from a split population duration model. J Health Econ 1994; 13: 213-30.

19. Roberts MJ, Samuelson L. An empirical analysis of dynamic, non-price competition in an oligopolistic industry. Rand J Econ 1988; 19:200-20.

20. Saffer $\mathrm{H}$, Chaloupka FJ. Tobacco advertising: economic theory and international evidence. Working Paper $n^{\circ}$ w6958. National bureau of economic research (NBER), 1999.

21. Norton $\varepsilon C$, Lindrooth RC, Ennett ST. Controlling for the endogeneity of peer substance use on adolescent alcohol and tobacco use. Health Econ $1998 ; 7: 439-53$

TIRÉS À PART 